\title{
A DATA-DRIVEN APPROACH FOR PREDICTING FAILURE SCENARIOS IN NUCLEAR SYSTEMS
}

\author{
Enrico Zio, Francesco Di Maio, Marco Stasi \\ Energy Department, Polytechnic of Milan \\ Via Ponzio 34/3, 20133 Milano, Italy \\ enrico.zio@polimi.it
}

\begin{abstract}
A data-driven approach is presented for the on-line identification of the system Failure Mode (FM) and the prediction of the available Recovery Time $(R T)$ during a failure scenario, i.e., the time remaining until the system can no longer perform its function in an irreversible manner. The FM identification and $R T$ prediction modules are linked in a general framework that recognizes the patterns of dynamic evolution of the process variables in the different system failure modes. When a new failure scenario develops, its evolution pattern is compared by fuzzy similarity analysis to a library of reference multidimensional trajectory patterns of process variables evolution; the failure mode of the developing scenario is identified by combining the modes of failure of the reference patterns, weighed by their similarity to the developing pattern; the similarity weights are then fed to the RT prediction module that estimates the time remaining before the developing trajectory pattern hits a failure threshold.

The approach is illustrated on failure scenarios of the Lead Bismuth Eutectic eXperimental Accelerator Driven System (LBE-XADS). The accident scenarios are classified in three different system failure modes, depending on the value reached by the diathermic oil secondary coolant temperature with respect to maximum and minimum safety threshold values set to avoid primary coolant thermal shocks and degradation of the oil physical and chemical properties.
\end{abstract}

Key Words: Failure Scenarios, Failure Mode Identification, Recovery Time Prediction, Data-driven Approach, Fuzzy Similarity Analysis, Nuclear Power Plant, Lead-Bismuth Eutectic eXperimental Accelerator Driven System (LBE-XADS). 


\section{Introduction}

The primary purpose of prognostics is to indicate whether the equipment of interest can perform its function throughout its lifetime; in case it cannot, it is a desirable feature of the prognostic methodology to identify the system Failure Mode (FM), i.e., the physical manner in which the failure occurs as defined by a safety variable crossing a pre-established threshold, and estimate the available Recovery Time (RT) during a failure scenario, i.e., the time remaining until the system can no longer perform its function in an irreversible manner.

The attractiveness of prognostics for Nuclear Power Plant (NPP) accident management comes from the fact that by predicting the evolution of the equipment dynamic state, it is possible to provide advanced warning for managing the accident and preparing the necessary corrective actions to maintain the equipment in productive operation while avoiding any undesired impact on safety [IAEA, 2003].

In case of an accident, or an initiating event that may develop into an accident, the plant personnel must perform various tasks before taking counteracting actions:

- Identification of the plant state: this diagnostic task aims at identifying the cause of the problem and the states of a number of parameters critical for the plant operation and safety;

- Prediction of the future development of the accident; this task consists in:

- the identification of the system FM, to recognize the corrective actions necessary to avoid the failure;

- the prediction of the available RT, which is the time remaining for taking the corrective actions.

- Planning of accident mitigation strategies, to be activated if safe control of the accident evolution was not successful.

NPP personnel have the capability to effectively manage a broad range of accidents; their successful management of complex accident behaviors requires that they detect the occurrence of 
the accident, determine the extent of challenge to plant safety, monitor the performance of active, passive, automatic and digital systems, select strategies to prevent or mitigate the safety challenge, implement the action strategies, and monitor their effectiveness. The capability to effectively carry out these tasks during an accident is influenced by the availability of timely and accurate plant status information, the awareness of the future accident progression towards the system FM and of the RT available for reaction. Poor decisions may be taken because of a misjudgment on the type of occurred accident and system progression towards failure and/or because of an assumed short time available for sorting out the information relevant to the plant status [Glasstone et al., 1998]; on the contrary, timely and correct decisions can prevent an event from developing into a severe accident or mitigate its undesired consequences.

Nowadays, operators are assisted by computer-based accident management support tools, since the complicated phenomena that take place in a NPP during an accident situation are more

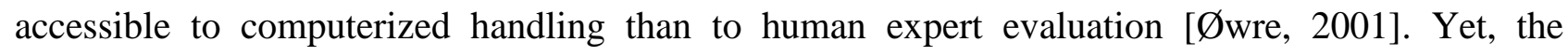
problem of what kind of decision support to provide to nuclear power plant operators, in particular during transients leading up to accidents, is far from trivial [NEA, 1992; EC, 1999; USNRC, 1999; IAEA, 2003].

Accident management support tools usually combine tracking and predictive simulators [IAEA, 2003]. The former ones monitor the plant status and provide calculated values also of those parameters that are not directly observable by the monitoring systems; predictive simulators deliver the FM and available RT for accident management.

Concerning the FM identification for a developing accidental scenario, it has been shown that the order and timing of the fault events occurring along an accident sequence, and the magnitude of the process variables at the time of event occurrence can be critical in determining the evolution of the accident and the FM the system is heading to [Aldemir et al., 2008]. The problem of inferring the system state from the measured parameters can be tackled by two general 
approaches: the model-based techniques [Willsky, 1976] and the model-free methods or data-driven approaches [Reifman, 1997; Marseguerra et al., 2006; Zio et al., 2009c]. Approaches to RT prediction can also be categorized broadly into model-based and data-driven [Chiang et al., 2001].

Model-based approaches attempt to incorporate physical models of the system into the estimations. However, the requirement of high computational speed for on line response necessarily leads to limited detail in the phenomena modeled, with consequent limited accuracy in the representation of the actual plant behavior [Berglund et al., 1995; Serrano et al., 1999]. On the contrary, data-driven techniques utilize monitored operational data related to system health. These techniques can typically be deployed quickly and cheaply, still providing wide coverage of system behavior; one should, however, be aware of the limitations of the data-driven approaches, which cannot be guaranteed to function properly in situations that are not included in the database used to train them. In this work, we will resort to a data-driven technique for the FM identification and RT prediction.

In general, data-driven approaches can be divided into two categories: statistical techniques (regression methods, ARMA models, etc.) and Artificial Intelligence (AI) techniques (neural networks, fuzzy logic systems, etc.).

With respect to AI techniques, Neural Networks (NNs) and Fuzzy Logic (FL) techniques have gained considerable attention in the past few years, due to their ability to deal with the uncertainties and non-linearities of the real processes, especially in abnormal conditions [Øwre, 2001]. Successful prediction models have been constructed based on Neural Networks [Barlett et al., 1992; Campolucci et al., 1999; Peel et al., 2008; Zio et al., 2008; Santosh et al., 2009] and Neuro-Fuzzy (NF) systems [Wang et al., 2004]. In spite of the recognized power of neural network modeling techniques, skepticism on their use in safety-critical applications relates to their black-box character which limits intuition with respect to the understanding of their performance [Wang et al., 2008]. 
An opportunity for increased transparency and openness of data-driven models is offered by fuzzy logic methods, which are increasingly proposed in modern control and diagnostic technologies. Based on the principles of Zadeh's fuzzy set theory, fuzzy logic provides a formal mathematical framework for dealing with the vagueness of everyday reasoning [Zadeh, 1965]. As opposed to binary reasoning based on ordinary set theory, within the fuzzy logic framework measurement uncertainty and estimation imprecision can be properly accommodated [Yuan et al., 1997; Zio et al., 2005].

The present work has the goal of enhancing the data-driven framework of RT prediction proposed in [Zio et al., 2009b], by including a module of system FM identification; the extension provides real-time information that is helpful for setting adequate corrective actions. The computational tool considers a set of multidimensional trajectory patterns arising from different system accident scenarios (hereafter called reference trajectory patterns) and uses a fuzzy-based, data-driven similarity analysis for identifying the FM and predicting the RT of a newly developing failure trajectory (hereafter called test trajectory pattern). The pattern matching process is based on a fuzzy evaluation of the distance between the signals of the multidimensional test pattern and the patterns of reference [Angstenberger, 2001]; the fuzzy distances between the reference patterns and the test one are combined to transform the multidimensional data into a one-dimensional similarity indicator, which is used for the identification of the FM and prediction of the available RT. To keep the focus, the analysis does not cover the identification of the type of faults occurred during the developing accidental scenario nor the distinction/identification of the effects of control actions/maneuvers or faults, which also determine deviations in the process variables. Future work will be directed towards the implementation of a fault detection and classification module for further improving the overall capabilities of the accident management support tool.

Dynamic failure scenarios of the Lead Bismuth Eutectic eXperimental Accelerator Driven System (LBE-XADS) with digital Instrumentation and Control (I\&C) [Cammi et al., 2006] are 
considered for the purpose of showing the feasibility of identifying the system FM and predicting the available RT. The analysis does not cover the study of the software and its possible failure modes, the benefits of fault tolerant features, the interactions of the software with the hardware and human components; eventually, these aspects will need to be addressed and included for the final implementation of the method in a qualified tool of accident management.

The paper is structured as follows. Section 2 provides a detailed description of the computational algorithm for the FM identification and RT prediction. Section 3 presents the mechanistic model of the LBE-XADS, with the description of the monitored signals. In Section 4, the results of the application of the approach to LBE-XADS failure scenarios are presented. Finally, some conclusions are drawn in Section 5.

\section{Methodology}

It is assumed that $N$ trajectories (reference trajectory patterns) are available, representative of the evolution of relevant process signals during randomly selected reference failure scenarios, ending in anyone of the possible system end states, which can be of failure or not. These trajectories last all the way to system failure, i.e., to the time when anyone of the signals reaches the threshold value defining the system FM beyond which the system loses its functionality, or to a fixed time horizon of observation of the failure process, $T$.

At any time, a developing multidimensional signal trajectory (test trajectory pattern) is compared for similarity with the $N$ multidimensional reference trajectory patterns stored in the database: the failure modes of these are used to identify the FM towards which the evolving transient is heading whereas their residual lifes are used to estimate the RT available.

Figure 1 shows a schematics of the computational framework in the general case of multidimensional trajectories of $Z$ monitored signals $f\left(x_{1}, x_{2}, \ldots, x_{Z}, t\right)$ at time $t$. 


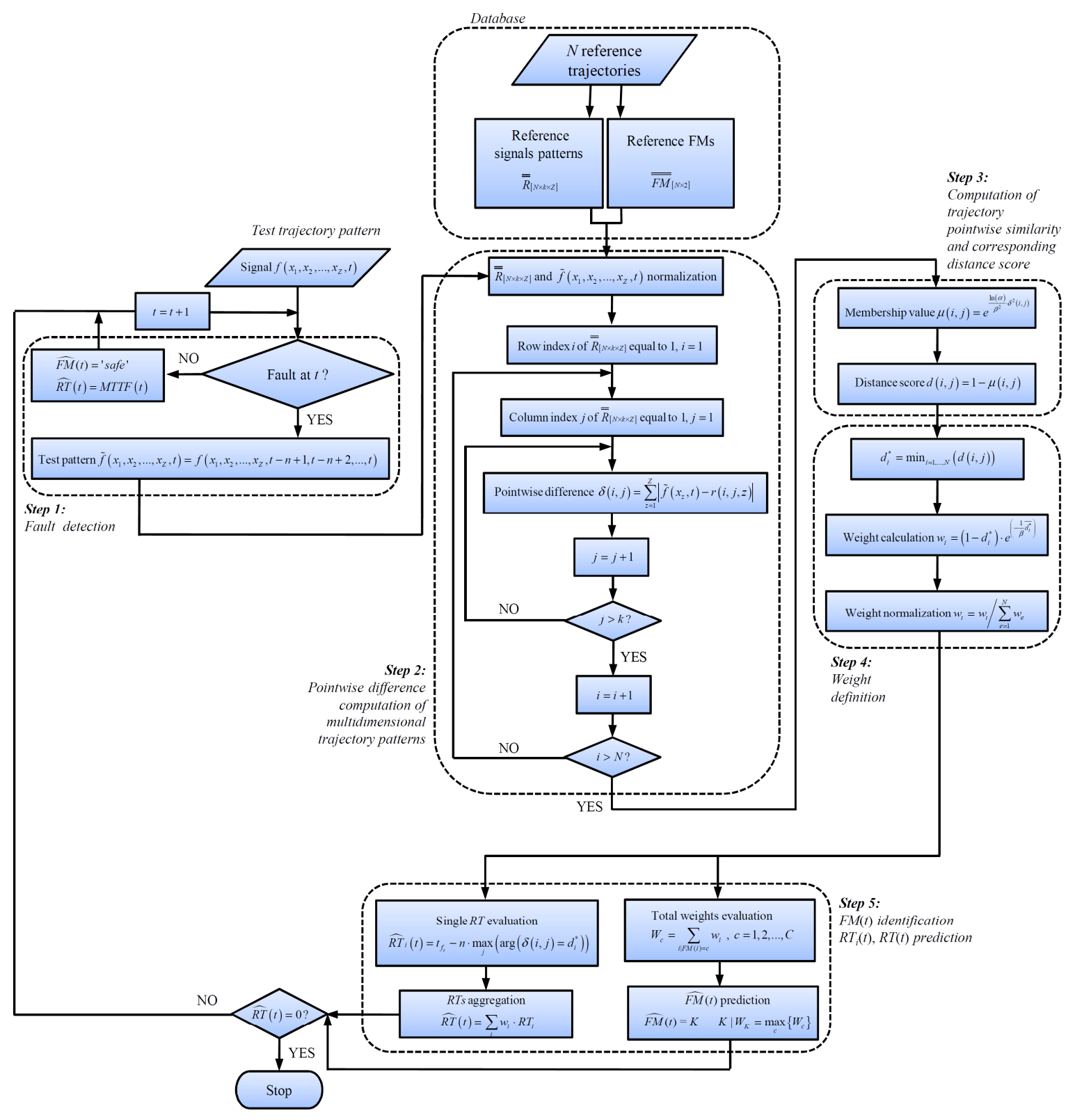

Figure 1 The flowchart of the fuzzy based data-driven approach

For illustration, the procedure is here followed step by step: the key modifications for FM identification with respect to the previous procedure for RT prediction [Zio et al., 2009a] are highlighted, together with the motivations behind it.

- Step 1: fault detection. The Z-dimensional trajectory of signals $f\left(x_{1}, x_{2}, \ldots, x_{Z}, t\right)$ is continuously monitored throughout the time horizon of observation $T$, starting from (discrete) time $t=1$; at each discrete time $t$, its values are recorded and appended to the matrix of the values collected at the previous time steps. 
For reasons which will become clear in the following, the database containing the information related to the reference trajectory patterns is organized in a failure mode matrix $\overline{\overline{F M}}_{[N \times 2]}$ and in a 3-D structure of process signals patterns $\overline{\bar{R}}_{[N \times k \times Z]}$. The failure mode matrix $\overline{\overline{F M}}_{[N \times 2]}$ contains the reference trajectories failure modes $F M_{i}=c, \quad c=1,2, \ldots, C$, $i=1,2, \ldots, N$, where $C$ is the maximum number of possible system FMs and $N$ is the number of the reference trajectories. The 3-D process signals patterns structure $\overline{\bar{R}}_{[N \times k \times Z]}$ contains the partitions of the reference trajectories, where $k=\frac{T}{n}$ and its generic element $r(i, j, z)$ is the projection on the $z$-th signal axis of $r(i, j)$ which is the $j$-th segment of length $n$ of values of the $i$-th reference trajectory, $i=1,2, \ldots, N, j=1,2, \ldots, k, z=1,2, \ldots, Z$, normalized in the range [0.2,0.8]. For clarity's sake, in Figures 2 and 3 a 2-D reference trajectory and its partition into 15 elements are shown, respectively (i.e., $Z=2$ and $k=15$ ).

As long as no abnormal signal deviation is detected, the system is qualified as working in nominal conditions, i.e., the Failure Mode $\widehat{F M}(t)$ is identified with the label corresponding to safe system, and the estimate $\widehat{R T}(t)$ of the available recovery time evaluated at the generic time $t$ is taken equal to the system Mean Time to Failure $\operatorname{MTTF}(t)$, obtained from the available recovery time $R T_{i}(t)$ of all the failure trajectories in the reference library:

$$
\widehat{R T}(t)=\operatorname{MTTF}(t)=\frac{1}{\left(\mid i \| t_{f_{i}}>t\right)} \sum_{i \mid t_{f_{i}}>t}\left(t_{f_{i}}-t\right)=\frac{1}{\left(\mid i \| t_{f_{i}}>t\right)} \sum_{i \mid t_{f_{i}}>t} R T_{i}(t)
$$

where $t_{f_{i}}$ is the time of system failure along the $i$-th reference trajectory (i.e., the time when the signal value exceeds the threshold beyond which the system loses its functionality), $\left(\mid i \| t_{f_{i}}>t\right)$ is the cardinality of the set of reference trajectories whose failure time is larger 
than $t$ and $R T_{i}(t)$ is their residual life starting from $t$. At the following time steps, the algorithm continues to update the estimates of $\widehat{R T}(t)=\operatorname{MTTF}(t)$, with the system in $\widehat{F M}(t)=$ 'safe' , until a fault is detected upon a deviation of the signal outside its range of allowed variability (which is a priori gauged with respect to the range of possible fluctuations in the signals); at this time, the algorithm for the identification of the FM and prediction of the available RT starts matching the similarity of the developing signal trajectory evolution to those in the reference library, combining their failure modes and failure times to provide an estimate of the system FM and available RT, respectively.
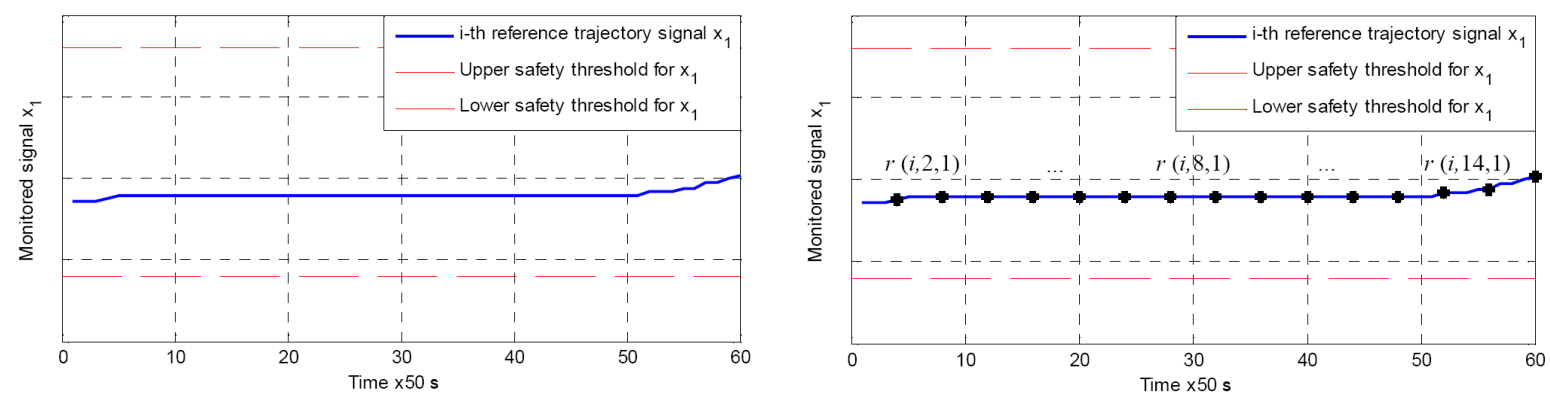

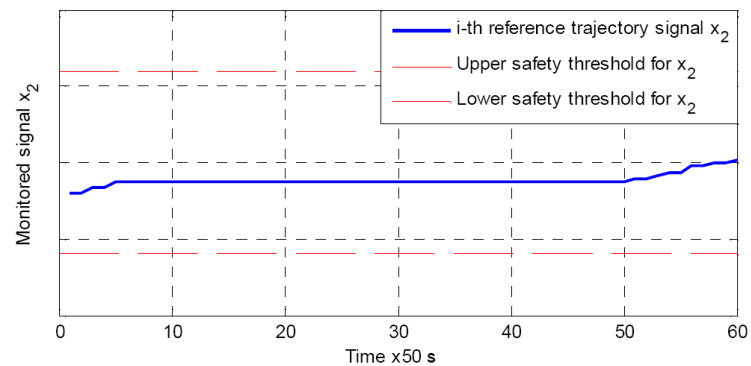

Figure 2 The two monitored signals of a safe bidimensional reference trajectory on a time horizon $T=3000 \mathrm{~s}$

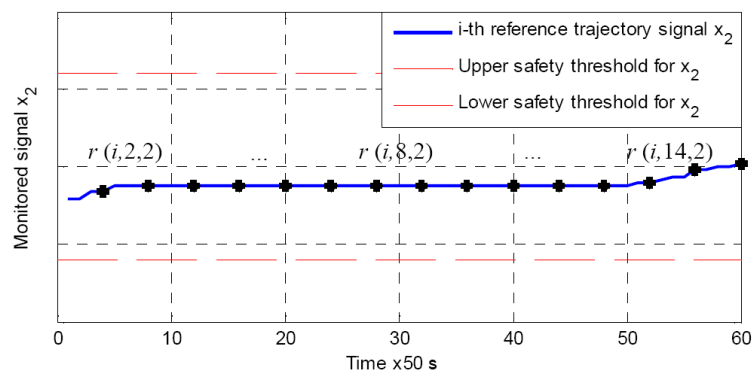

Figure 3 The two monitored signals of Figure 2 partitioned into $k=15$ segments of length $n=3000 / 15=200 \mathrm{~s}$, $j=1,2, \ldots, 15$

Step 2: pointwise difference computation of multidimensional trajectory patterns. At the current time $t$, the latest $n$-long segment of values of the test trajectory pattern $\tilde{f}\left(x_{1}, x_{2}, \ldots, x_{Z}, t\right)=f\left(x_{1}, x_{2}, \ldots, x_{Z}, t-n+1, t-n+2, \ldots, t\right)$ is normalized within an arbitrary chose range (a common choice is $[0.2,0.8]$ which avoids numerical problems, e.g. arising from the zero value in a range centered at the origin). The pointwise difference $\delta(\bullet)$ 
between the $n \cdot Z$ values of pattern $\tilde{f}\left(x_{1}, x_{2}, \ldots, x_{Z}, t\right)$ and those of the generic reference trajectory segment $r(i, j, z)$, is computed:

$$
\delta(i, j)=\sum_{z=1}^{Z}\left|\tilde{f}\left(x_{z}, t\right)-r(i, j, z)\right|, i=1,2, \ldots, N, j=1,2, \ldots, k, z=1,2, \ldots, Z
$$

The matrix $\overline{\bar{\delta}}_{[N \times k]}$ contains the difference measures $\delta(i, j)$ between all $n$-long segments of the Z-dimensional reference trajectories and the test trajectory pattern of the monitored signals.

Step 3: computation of trajectory pointwise similarity and corresponding distance score.

To allow for a gradual transition in the similarity measure [Binaghi et al., 1993; Joentgen et al., 1999], the pointwise difference between two trajectories is evaluated with reference to an "approximately zero" fuzzy set (FS) specified by a function which maps the elements $\delta(i, j)$ of the difference matrix $\overline{\bar{\delta}}_{[N \times k]}$ into their values $\mu(i, j)$ of membership to the condition of "approximately zero". The distance score $d(i, j)$ between two trajectory segments is then computed as:

$$
d(i, j)=1-\mu(i, j), i=1,2, \ldots, N, j=1,2, \ldots, k
$$

In the application illustrated in this work, the following bell-shaped function is used [Dubois et al., 1988]:

$$
\mu(i, j)=e^{-\left(\frac{-\ln (\alpha)}{\beta^{2}} \delta^{2}(i, j)\right)}
$$

The arbitrary parameters $\alpha$ and $\beta$ can be set by the analyst to shape the desired interpretation of similarity into the fuzzy set: the larger the value of the ratio $\frac{-\ln (\alpha)}{\beta^{2}}$, the 
narrower the fuzzy set and the stronger the definition of similarity [Zio et al., 2009a]. Other common membership functions can be used, e.g. triangular and trapezoidal.

\section{- Step 4: weight definition.}

All failure trajectories in the reference library can bring useful information for determining the available RT and predicting the system FM of the trajectory currently developing; on the other hand, those segments of the reference trajectories which are most similar to the most recent segment of length $n$ of the currently developing failure trajectory should be more informative in the extrapolation of the occurring trajectory to failure to identify $\widehat{F M}(t)$ and estimate $\widehat{R T}(t)$. To account for this, $\widehat{F M}(t)$ is identified as:

$$
\widehat{F M}(t)=K, \text { with } K \mid W_{K}=\max _{c}\left\{W_{c}\right\}, \quad c=1,2, \ldots, C
$$

where $W_{c}=\sum_{i \mid F M_{i}=c} w_{i}, \quad i=1,2, \ldots, N, \quad c=1,2, \ldots, C$, is the sum of the weights $w_{i}$ of those reference trajectories whose $F M_{i}=c$ and $\widehat{R T}(t)$ is estimated as a similarity-weighted sum of the $R T_{i}(t)$ :

$$
\widehat{R T}(t)=\sum_{i f_{f_{i}}>t} w_{i} \cdot R T_{i}(t), i=1,2, \ldots, N
$$

To assign the weight $w_{i}$, the minimum distance $d_{i}^{*}$ along the $i$-th row of the matrix of Eq. (3) is first identified:

$$
d_{i}^{*}=\min _{j=1, \ldots, k} d(i, j), i=1,2, \ldots, N
$$

The weight $w_{i}$ is then computed as:

$$
w_{i}=\left(1-d_{i}^{*}\right) \cdot e^{\left(-\frac{1}{\beta} d_{i}^{*}\right)}, i=1,2, \ldots, N
$$


and normalized:

$$
w_{i}=w_{i} / \sum_{e=1}^{N} w_{e}
$$

Note that the smaller the minimum distance the larger the weight given to the $i$-th trajectory [Zio et al., 2009].

Step 5: FM(t) identification; $R T_{i}(t), R T(t)$ prediction. The $i$-th reference trajectory of the library, $i=1,2, \ldots, N$, partakes in the FM identification by voting for the class $c$ it belongs to (Eq. (5)), $c=1,2, \ldots, C$; depending on its similarity to the developing trajectory, its vote is weighed by the value $w_{i}$ defined in Eq. (9). The most voted class corresponds to the $K$-th class that labels $\widehat{F M}(t)$, providing the identification of the FM for the test trajectory at time $t$.

With respect to the generic $i$-th trajectory in the library for which $t_{f_{i}}>t$, the value $R T_{i}(t)$ is determined as:

$$
R T_{i}(t)=t_{f_{i}}-t_{j_{M}}, i=1,2, \ldots, N
$$

where $t_{j_{M}}=n \cdot \max _{j}\left(\arg \left(\delta(i, j)=d_{i}^{*}\right)\right)$ is the final time index of the latest-in-life segment of the $i$-th trajectory among those with minimum distance $d_{i}^{*}$ from the developing test trajectory ( $n$ is the test trajectory pattern length and $\max _{j}\left(\arg \left(\delta(i, j)=d_{i}^{*}\right)\right)$ gives the largest column index $j$ of $r(i, \bullet)$ whose element is equal to $\left.d_{i}^{*}\right)$. Thus, $R T_{i}(t)$ is the time available before reaching the failure threshold on the reference trajectory starting from the end time of the latest-in-life segment of minimum distance from the developing trajectory (Figure 4). 


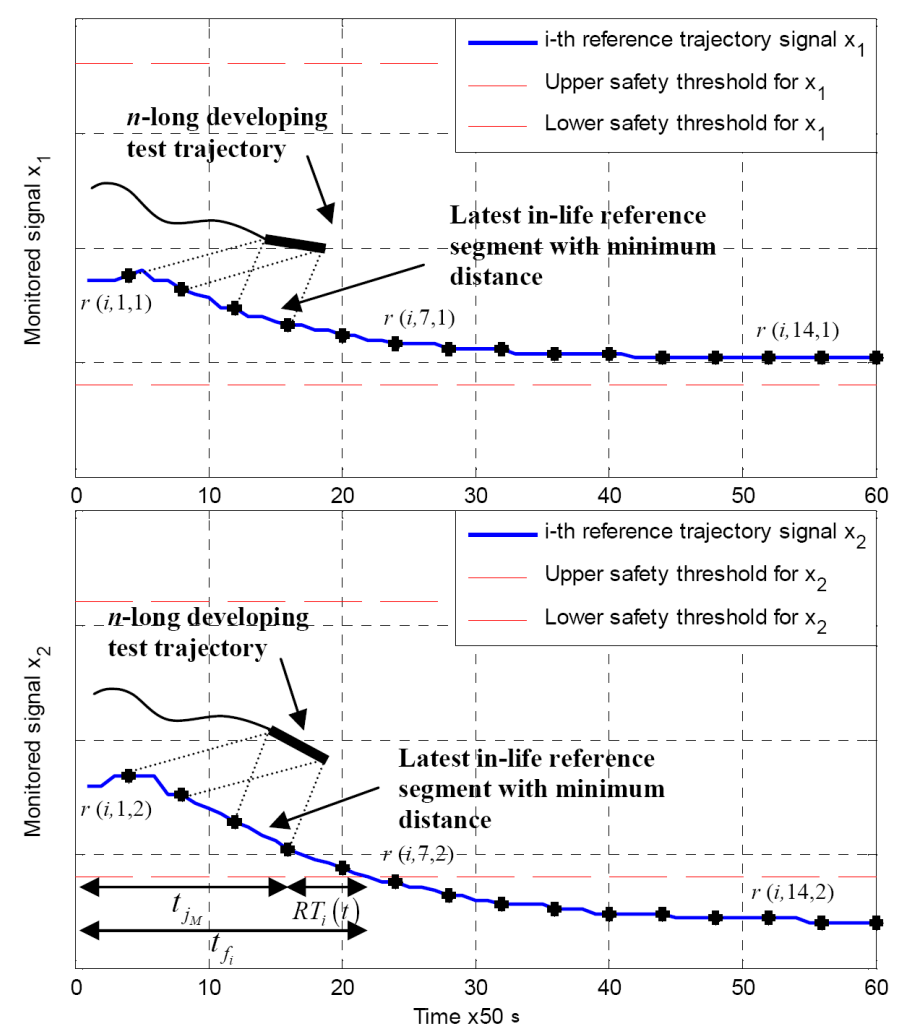

Figure 4 The $R T_{i}(t)$ for the $i$-th bidimensional reference trajectory starts from the end time of the latest-in-life segment of minimum distance from the occurring trajectory

This allows a conservative RT estimation, biased towards "pessimistic" predictions of the available RT, because in the case that more than one segment along the $i$-th reference trajectory is closest to the developing test trajectory, the latest one is taken, i.e., the one closest to failure.

Then, the estimate $\widehat{R T}(t)$ of the remaining useful life along the developing trajectory is simply computed as in Eq. (6), with weights $w_{i}$ evaluated by Eq. (9).

\section{The LBE-XADS}

The case study considered is the same as in [Zio et al., 2009a], concerning failure trajectories in the Lead-Bismuth Eutectic eXperimental Accelerator Driven System (LBE-XADS), a sub-critical, fast reactor in which the fission process for providing thermal power $P(t)$ is sustained 
by an external neutron source through spallation reaction by a proton beam $Q(t)$ accelerated by a synchrotron on a lead-bismuth eutectic target [Bowman et al., 1992; Carminati et al., 1993; Rubbia et al., 1995; Van Tuyle et al., 1993; Venneri et al., 1993]. For completeness of the information in the paper, the physical description of the LBE-XADS and of the failures considered are here repeated, with reference to the simplified scheme of the plant of Figure 5. The primary cooling system is of pool-type with Lead-Bismuth Eutectic (LBE) liquid metal coolant leaving the top of the core, at full power nominal conditions, at temperature equal to $400{ }^{\circ} \mathrm{C}$ and then re-entering the core from the bottom through the down-comer at a temperature of $300{ }^{\circ} \mathrm{C}$. The average in-core temperature of the $\operatorname{LBE} T_{L B}^{a v, C}$ is taken as the mean of the entrance and exit temperatures. The secondary cooling system is a flow of an organic diathermic oil at $290-320{ }^{\circ} \mathrm{C}$, at full power conditions. Cooling of the diathermic oil in each loop is obtained through an air flow $\Gamma_{a}(t)$ provided by three air coolers connected in series.

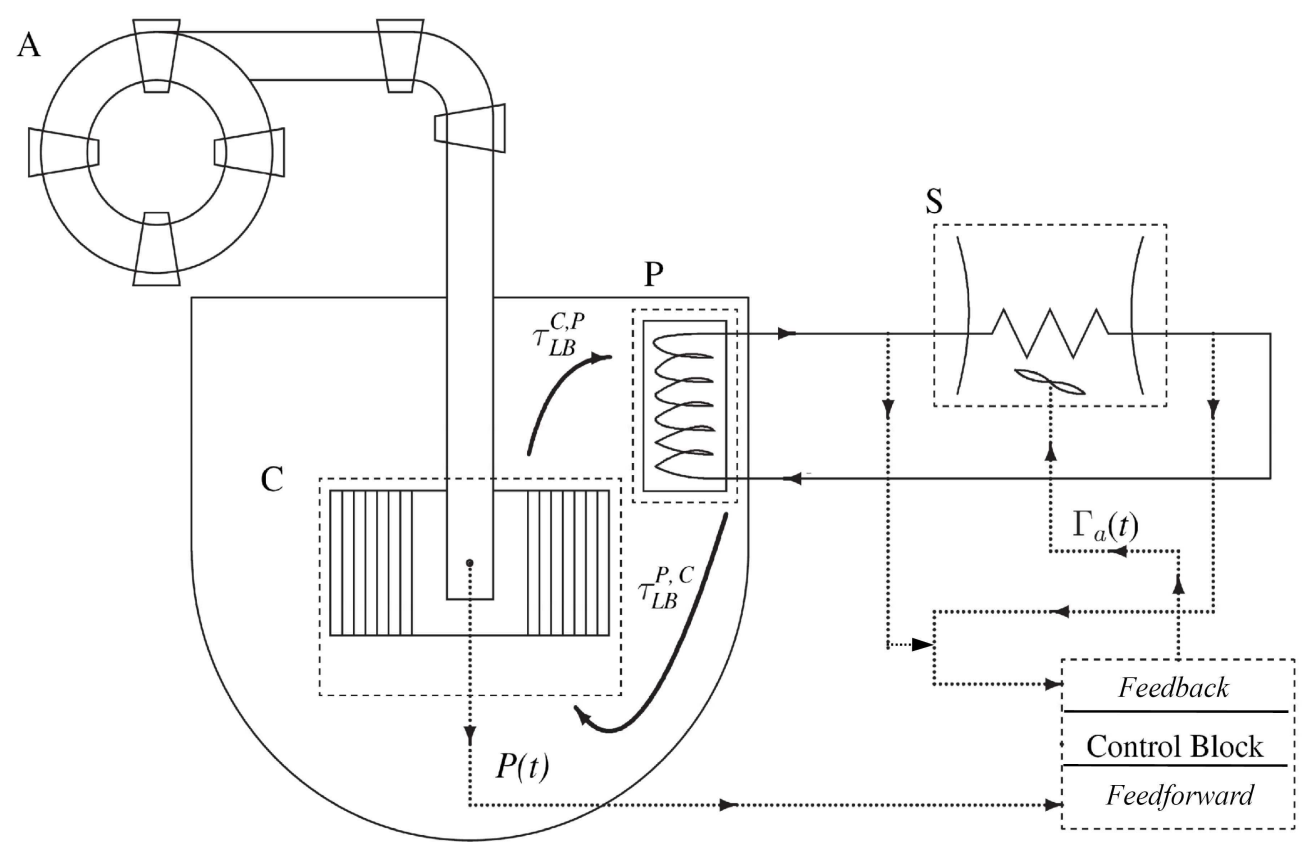

Figure 5 LBE-XADS simplified schematics. $A=$ Accelerator; $C=$ core; $P$ = primary heat exchanger; $S=$ secondary heat exchanger 
A dedicated, dynamic simulation model has been implemented in SIMULINK for providing a simplified, lumped and zero-dimensional description of the coupled neutronic and thermohydraulic evolution of the system [Cammi et al., 2006]. The model allows the simulation of the system controlled dynamics as well as of the free dynamics when the control module is deactivated and the air cooler flow is kept constant.

Both feedforward and feedback digital control schemes have been adopted for the operation of the system. The control is set to keep a steady state value of approximately $300{ }^{\circ} \mathrm{C}$ of the average temperature of the diathermic oil $T_{o}^{a v, S}$ : this value represents the optimal working point of the diathermic oil at the steady state, full nominal power of $80 \mathrm{MWth}$.

On the contrary, an oil temperature beyond the upper threshold $T_{o}^{\text {th, } u}=340{ }^{\circ} \mathrm{C}$ would lead to degradation of its physical and chemical properties, whereas a temperature below the lower threshold $T_{o}^{\text {th,l }}=280{ }^{\circ} \mathrm{C}$ could result in thermal shocks for the primary fluid and, eventually, for the structural components [Cammi et al., 2006]. Conservatively, no dependence on the duration of exposition to temperatures beyond the threshold values has been assumed: in other words, the system is considered to fail at such temperatures regardless of the time during which it exceeds the thresholds.

Multiple component failures can occur during the system life. To simulate this, the model has been embedded within a Monte Carlo (MC) sampling procedure for injecting faults at random times and of random magnitudes. Samples of component failures are drawn within a time horizon of 3000 s. The set of faults considered are:

- The PID controller fails stuck at time $t_{1}$ with a random flow rate output value $m_{1}$ sampled from a uniform distribution in $[0,797] \mathrm{Kg} / \mathrm{s}$.

- The air coolers fail stuck at time $t_{2}$ in a random position that provides a corresponding air flow mass $m_{2}$ uniformly distributed in $[0,1000] \mathrm{Kg} / \mathrm{s}$. 
- The feedforward controller fails stuck at time $t_{3}$ with a corresponding flow rate value $m_{3}$ uniformly distributed in $[0,797] \mathrm{Kg} / \mathrm{s}$.

- The communication between air coolers actuators and PID controller fails at time $t_{4}$ so that the PID is provided with the same input value of the previous time step.

The first three faults are applicable to both analog and digital systems, whereas the last one is typical of digital systems. Furthermore, the fault magnitude probability distributions are assumed to be uniform, even if the components may more likely fail in a certain mode than in others. This includes also rare multiple events in the set of failure scenarios and further tests the robustness of the FM identification and RT prediction.

The sequence of multiple failures is generated by sampling the first failure time from the uniform distribution $[0,3000] \mathrm{s}$ and the successive failure times from the conditional distributions, uniform from the last sampled time to $3000 \mathrm{~s}$. This assumption is conservative, favoring larger numbers of failures in the sequence.

The evolution of the failure scenarios may lead to three different Failure Modes (FMs), within the mission time of $3000 \mathrm{~s}$, labeled with numbers from 1 to 3 :

$\mathrm{FM}=1 . \quad$ Low-temperature failure mode $\left(T_{o}^{a v, S}<T_{o}^{t h, l}\right)$

$\mathrm{FM}=2 . \quad$ Safe mode $\left(T_{o}^{t h, l}<T_{o}^{a v, S}<T_{o}^{t h, u}\right)$

$\mathrm{FM}=3 . \quad$ High-temperature failure mode $\left(T_{o}^{a v, S}>T_{o}^{t h, u}\right)$

The following three signals are taken for the identification of the system FM and the prediction of the available RT:

- Mean in-core LBE temperature, $T_{L B}^{a v, C}$

- Mean oil temperature of the secondary heat exchanger hot side, $T_{o}^{a v, S}$

- Mean air flow rate at the secondary heat exchanger cold side, $\Gamma_{a}(t)$ 
Notice that the sampling of the fault events here undertaken is not intended to reproduce the actual stochastic failure behavior of the system components; rather, the choices and hypotheses for modeling the faults (i.e., the time horizon of the analysis, the number and typology of faults, the distributions of failure times and magnitudes) have been arbitrarily made with the aim of favoring multiple failures. Further, the components considered subjected to fault and the fault mechanisms are not intended to provide a comprehensive description of the system fault behavior but are only taken as exemplary and used for generating the dynamic failure scenarios to be used as reference and test patterns.

\section{Results}

\subsection{Application of the procedure for FM(t) identification and $R T(t)$ prediction}

A total of $N=6400$ reference failure scenarios have been simulated, differing in faulty components, times of faults occurrence and faults magnitudes. For each scenario, $Z=2$ evolution trajectories of the process variables $T_{L B}^{a v, C}$ and $T_{o}^{a v, S}$ are considered. The database of reference trajectories is organized in the signals patterns structure $\overline{\bar{R}}_{[N \times k \times Z]}$, where $k=\frac{T}{n}=60$; the generic element $r(i, j, z)$ of the reference structure is compared for similarity with the $z$-th signal of the test trajectory pattern containing the values of the latest 50 time steps of the trajectory. The matrix $\overline{\overline{F M}}_{[N \times 2]}$ contains the $N=6400$ reference trajectories failure modes $F M_{i}=c, \quad c=1,2,3$, $i=1,2, \ldots, 6400$.

For each of the test trajectories, the procedural steps 1-5 of Section 2 are performed.

As an example, for the 2-D test pattern trajectories of Table 1 , the $\widehat{F M}(t)$ identifications based on trajectory segments of $n=50 \mathrm{~s}$ are plotted in the upper subplots of Figures 6-10; for all the trajectories, $F M(t)$ is defined as 'safe' $(F M=2)$ until the first fault occurs and the pattern 
similarity matching starts. In the Figures, the bold vertical line indicates the time of diathermic oil temperature threshold exceedance, i.e., the time the system fails if effective recovery actions are not successfully completed before then.

\begin{tabular}{|l|c|c|c|c|c|c|c|}
\cline { 2 - 8 } \multicolumn{1}{c|}{} & $\boldsymbol{t}_{\mathbf{1}}[\mathrm{s}]$ & $\boldsymbol{m}_{\mathbf{1}}[\mathrm{Kg} / \mathrm{s}]$ & $\boldsymbol{t}_{\mathbf{2}}[\mathrm{s}]$ & $\boldsymbol{m}_{\mathbf{2}}[\mathrm{Kg} / \mathrm{s}]$ & $\boldsymbol{t}_{\mathbf{3}}[\mathrm{s}]$ & $\boldsymbol{m}_{\mathbf{3}}[\mathrm{Kg} / \mathrm{s}]$ & $\boldsymbol{t}_{\mathbf{4}}[\mathrm{s}]$ \\
\hline $\begin{array}{l}\text { Trajectory belonging to the low- } \\
\text { temperature failure mode (Fig. 6) }\end{array}$ & 245 & 426 & $/$ & $/$ & $/$ & $/$ & 388 \\
\hline $\begin{array}{l}\text { Trajectory belonging to the low- } \\
\text { temperature failure mode (Fig. 7) }\end{array}$ & 1610 & 667 & 1290 & 962 & 2732 & 240 & 2845 \\
\hline $\begin{array}{l}\text { Trajectory belonging to the high- } \\
\text { temperature failure mode (Fig. 8) }\end{array}$ & 2933 & 492 & 2731 & 358 & 2988 & 713 & 2156 \\
\hline $\begin{array}{l}\text { Trajectory belonging to the high- } \\
\text { temperature failure mode (Fig. 9) }\end{array}$ & 1507 & 560 & 1382 & 551 & 1611 & 339 & 153 \\
\hline $\begin{array}{l}\text { Trajectory which does not exceed } \\
\text { any safety threshold value (Fig.10) }\end{array}$ & 2828 & 524 & 2722 & 72 & 2085 & 87 & 2066 \\
\hline
\end{tabular}

Table 1 Times and magnitudes of faults occurring during the accidental scenarios considered in Figures 6-10

The estimates of the $\operatorname{MTTF}(t)$ are plotted in the lower subplots of Figures 6-10, in thin continuous lines with the bars of one standard deviation of the samples $\left(t_{f_{i}}-t \mid t_{f_{i}}>t\right)$, where $t_{f_{i}}$ is the time at which the diathermic oil temperature profile of the $i$-th reference trajectory exceeds either thresholds $T_{o}^{t h, u}$ or $T_{o}^{t h, l}$, with corresponding system loss of functionality. The $\widehat{R T}(t)$ estimates, also obtained based on trajectory segments of $n=50 \mathrm{~s}$, are plotted in bold circles; at the beginning of the test trajectories, the predictions match the $\operatorname{MTTF}(t)$; then, once a component failure is detected, the $\widehat{R T}(t)$ estimates move away from the $\operatorname{MTTF}(t)$ values towards the real $R T(t)$ (dashed thick line). Notice that none of the $\widehat{R T}(t)$ estimates exceeds the actual failure time. 


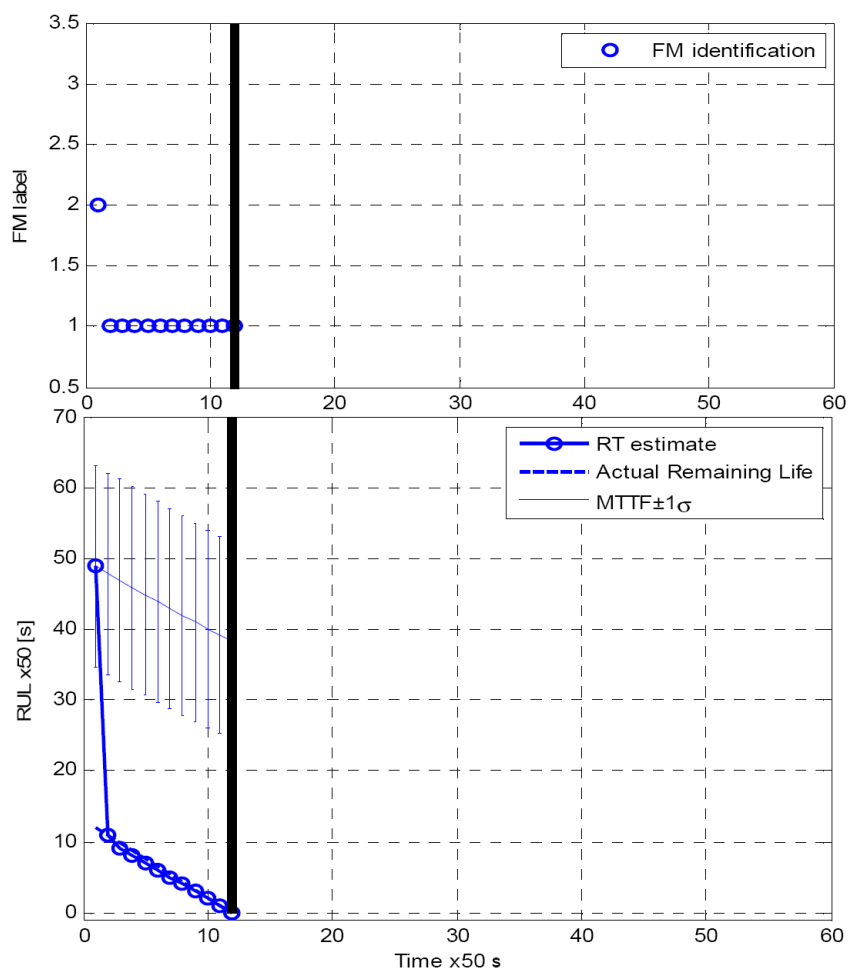

Figure 6 FM identification (top) and RT estimation (bottom) for a trajectory belonging to the low-temperature failure mode $(\mathrm{FM}=1)$

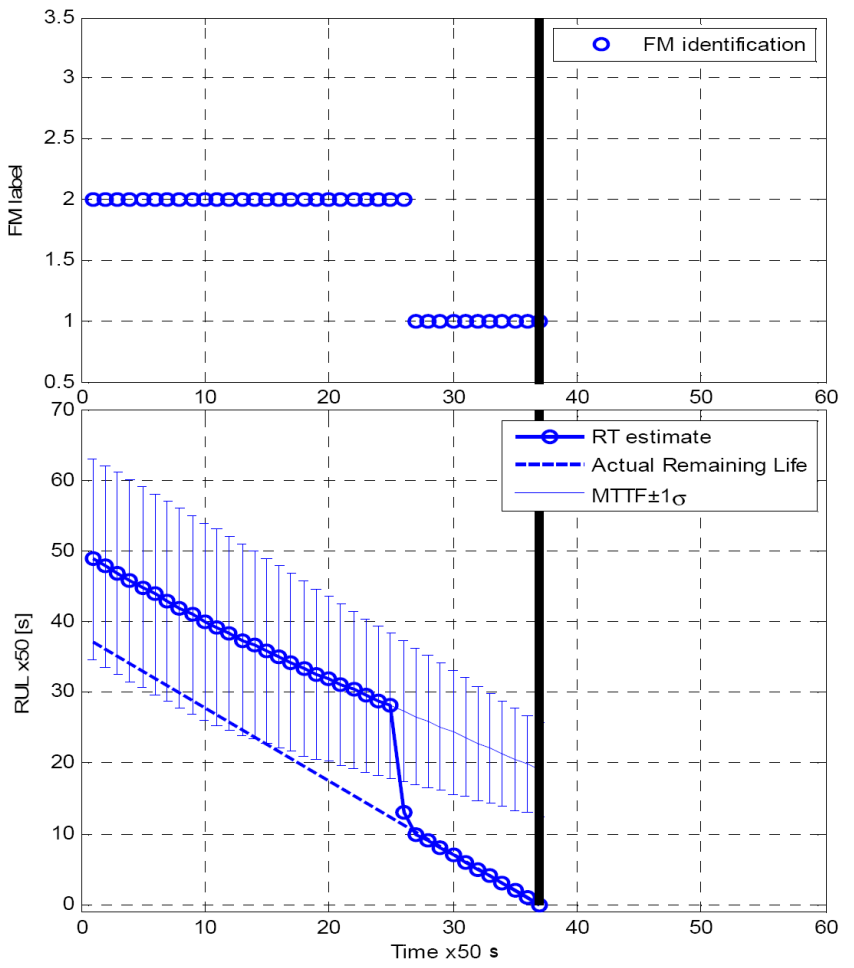

Figure 7 FM identification (top) and RT estimation (bottom) for a trajectory belonging to the low-temperature failure mode $(\mathbf{F M}=1)$

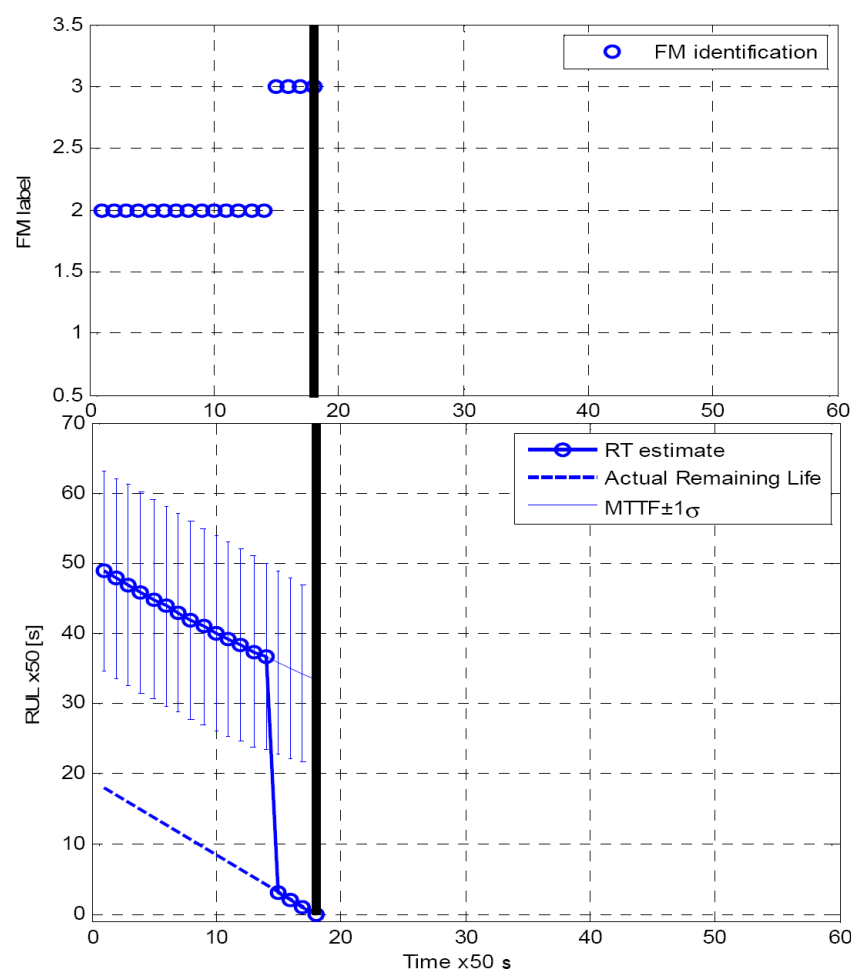

Figure 8 FM identification (top) and RT estimation (bottom) for a trajectory belonging to the high-temperature failure $\operatorname{mode}(\mathbf{F M}=3)$
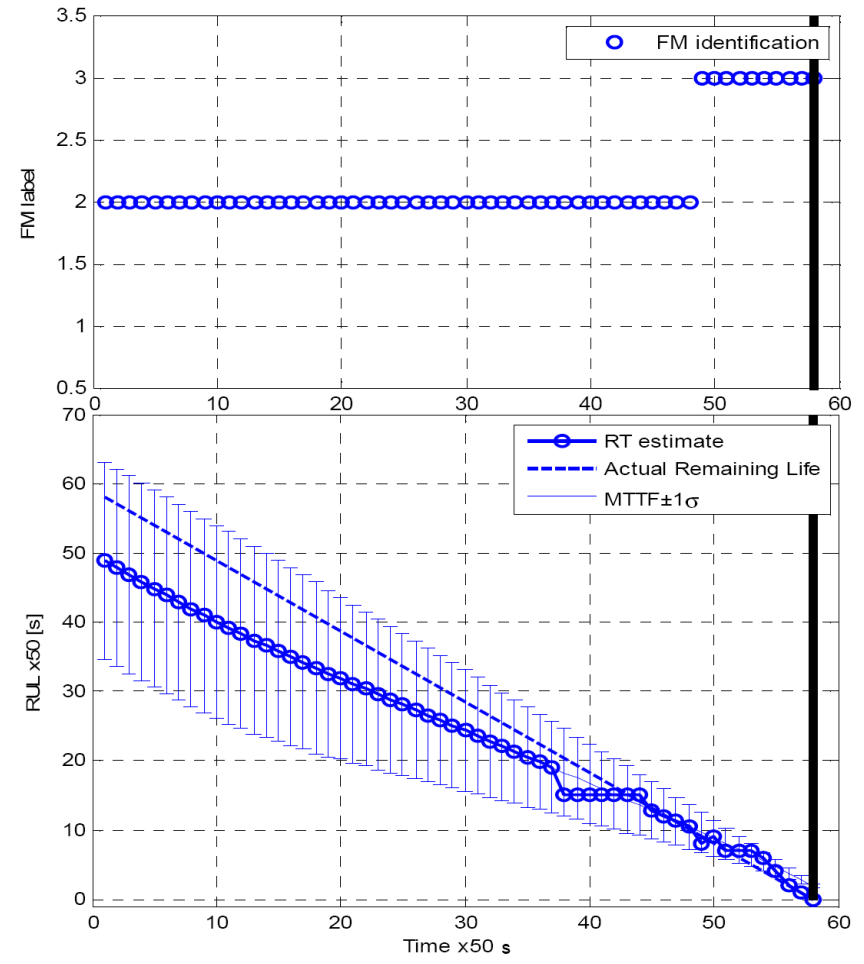

Figure 9 FM identification (top) and RT estimation (bottom) for a trajectory belonging to the high-temperature failure mode $(F M=3)$ 


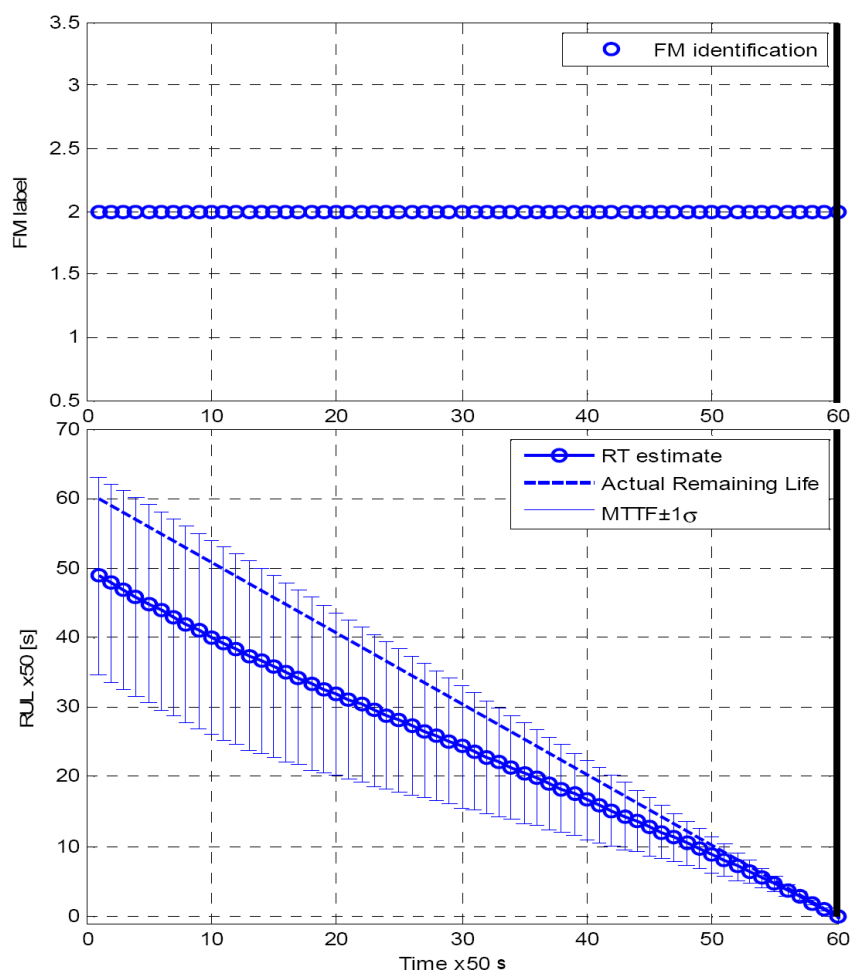

Figure 10 FM identification (top) and RT estimation (bottom) for a trajectory which does not exceed any safety threshold value, although a sequence of faults has occurred (FM=2)

\subsection{Performance of the FM(t) identification and $R T(t)$ prediction procedure}

The performance of the method for identifying the FM and predicting the available RT has been verified on a batch of $P=128$ multidimensional test trajectories, different from the reference ones.

Concerning the performance of the FM identification, the fraction of correct identifications over the total number $P$ of test trajectories tested is shown in Figure 11, in correspondence of three different prediction times $T_{10 \%}, T_{50 \%}$ and $T_{90 \%}$ corresponding to the time instants after $10 \%, 50 \%$ and $90 \%$ of the evolution of the test trajectory patterns, respectively; the performance increases as the developing trajectories approach the end of their lives, reaching a value of $86 \%$ at $T_{90 \%}$; at this time, the classification performance can be considered satisfactory, e.g. in comparison with the results of other methodologies applied on the same literature case study [Zio et al., 2009c]. 


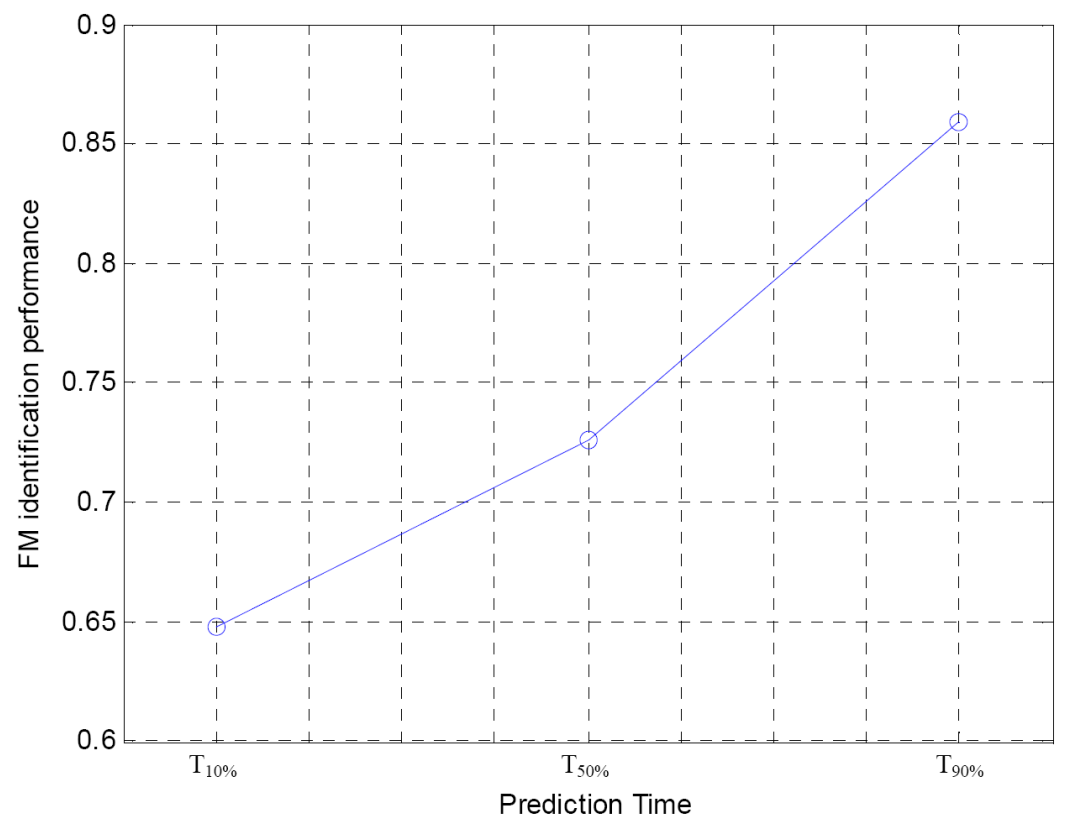

Figure 11 FM identification performance evaluated at times $T_{10 \%}, T_{50 \%}$ and $T_{90 \%}$ over the 128 test trajectories

The accuracy of the RT prediction at any time $t$ is expressed through the relative error (RE) between the estimate $\widehat{R T}(t)$ and its true value $R T(t)$. To globally quantify the performance of the procedure over the batch of trajectories, the mean RE at time $t$ has been evaluated:

$$
\overline{R E}(t)=\frac{1}{P} \sum_{p=1}^{P} \frac{\left|\widehat{R T}_{p}(t)-R T_{p}(t)\right|}{R T_{p}(t)}
$$

where $R T_{p}(t)$ is the actual available recovery time at time $t$ of test pattern $p$, and $\widehat{R T}_{p}(t)$ its estimate, $p=1,2, \ldots, P$.

Figure 12 shows the empirical probability density function of the RT mean relative error. The distribution is skewed towards small error values with mean and median equal to 0.09 and 0.04 , thus proving that the procedure most frequently makes small relative estimation errors. 


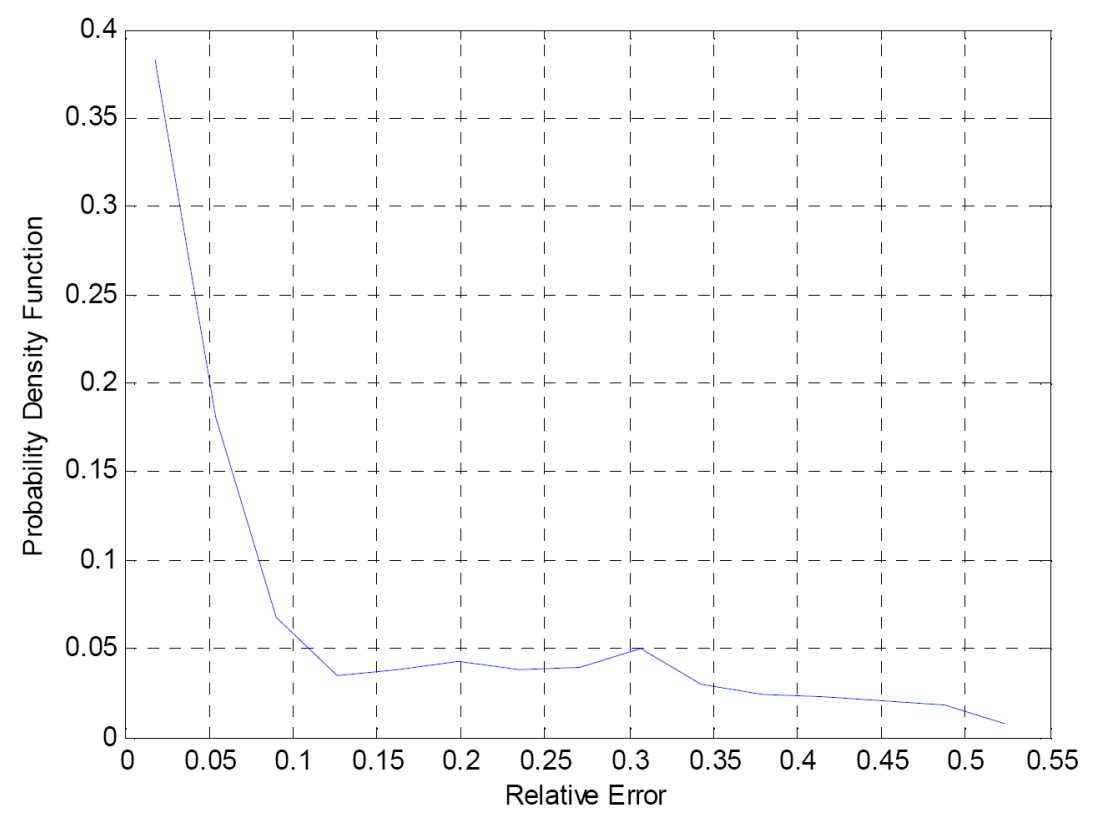

Figure 12 Empirical probability density function of the RE for the 128 test trajectories

Figure 13 and Figure 14 offer further insights on the RT relative error behavior and its decrease along the test trajectory life time: the former one shows the $\overline{R E}(t)$ in correspondence of three different prediction time instants $T_{10 \%}, T_{50 \%}$ and $T_{90 \%}$, i.e., after $10 \%, 50 \%$ and $90 \%$ of the evolution of the test trajectory patterns, respectively; the latter one reports the associated boxplots for the same three representative times. The accuracy of the RT estimation is seen to increase along the test trajectory patterns evolution: halfway $\left(T_{50 \%}\right), 75 \%$ of the relative errors are smaller than 0.1 ; towards the end $\left(T_{90 \%}\right), 98 \%$ of the relative errors are smaller than $0.1,75 \%$ are smaller than 0.05 and the value of the median of the distribution narrows down to that of the empirical probability density function of Figure 12. 


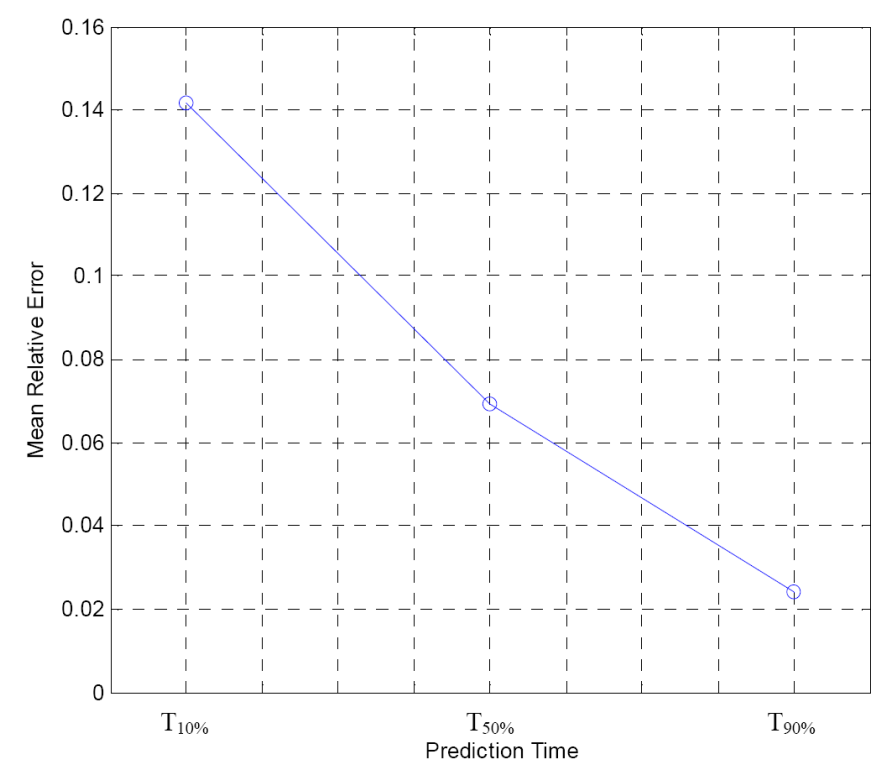

Figure 13 RT mean relative error evaluated at times $\boldsymbol{T}_{10 \%}$, $T_{50 \%}$ and $T_{90 \%}$ over the 128 test trajectories

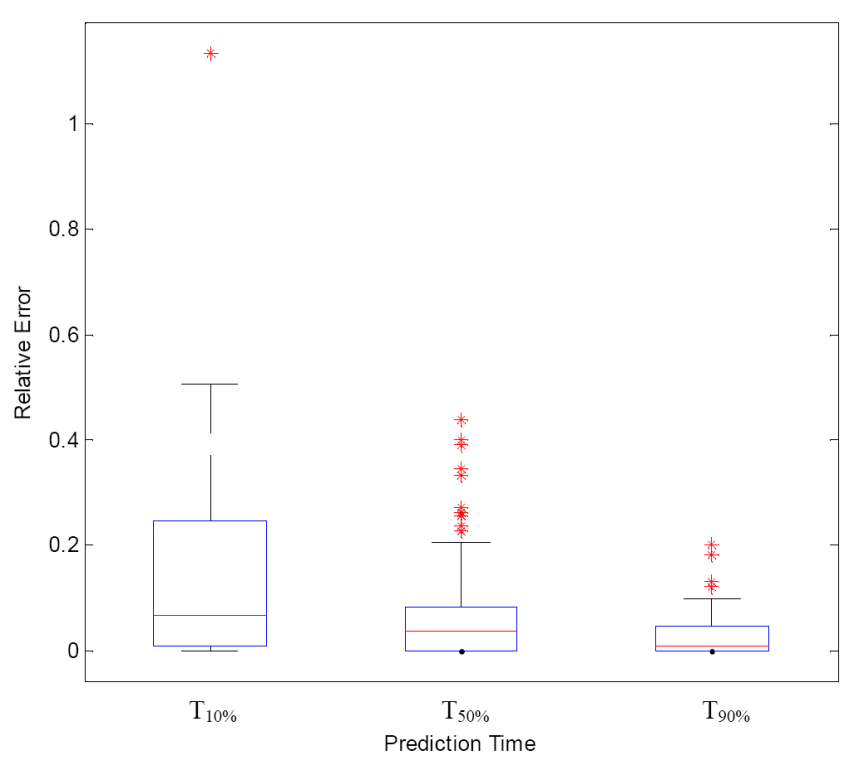

Figure 14 Boxplots of the relative errors evaluated at times $T_{10 \%}, T_{50 \%}$ and $T_{90 \%}$ over the 128 test trajectories

Finally, the computational time required by the procedure for one complete test trajectory of $3000 \mathrm{~s}$ is approximately $150 \mathrm{~s}$ on an Intel $^{\circledR}$ Core 2 Duo of $1.83 \mathrm{GHz}$, resulting in a $0.05 \mathrm{~s}$ time requested for prognosing on $1 \mathrm{~s}$ of trajectory evolution.

\section{Conclusions}

A data-driven similarity-based approach for identifying the Failure Mode (FM) of a system and predicting the available Recovery Time (RT) has been presented. The computational tool developed could be embedded in an operator support system for emergency accident management for timely and correct decisions on how to prevent an event from developing into a severe accident or mitigate its undesired consequences.

The approach considers the information carried out by multiple signals of multidimensional trajectories. Data from different transient failure scenarios are used to create a library of reference patterns of evolution. For identifying the FM towards which the system evolution is heading and predicting the available RT of a test pattern, its evolution data are matched to the reference patterns in the library within a multidimensional fuzzy pointwise similarity setting; the information from the 
reference patterns, i.e. their failure modes and residual life times, is combined based on the assessed similarity to provide the FM identification and RT prediction.

A number of fault scenarios in the Lead Bismuth Eutectic eXperimental Accelerator Driven System (LBE-XADS) have been analyzed by the proposed approach, with satisfactory results in terms of both accuracy and speed of computing.

\section{Acknowledgments}

The authors are thankful to the anonymous referees for the critical contribution, which has stimulated a significant improvement of the paper.

\section{References}

[Aldemir et al., 2008] T. Aldemir, S. Guarro, J. Kirschenbaum, D. Mandelli, L.A. Mangan, P. Bucci, M. Yau, B. Johnson, C. Elks, E. Ekici, M.P. Stovsky, D.W. Miller, X. Sun, S.A. Arndt, Q. Nguyen, J, Dion, A Benchmark Implementation of Two Dynamic Methodologies for the Reliability Modeling of Digital Instrumentation and Control Systems (NUREG/CR-6985).

[Angstenberger, 2001] Angstenberger, L., Dynamic Fuzzy Pattern Recognition, International Series in Intelligent Technologies, 17, Kluwer Academic Publishers, 2001.

[Barlett et al., 1992] Barlett, E.B., Uhrig, R.E., Nuclear Power Plant Status Diagnostics Using an Artificial Neural Network, Nuclear Technology, 97, 1992.

[Binaghi et al., 1993] Binaghi, E., Della Ventura, A., Rampini, A., Schettini, R. (1993) Fuzzy Reasoning Approach to Similarity Evaluation in Image Analysis. International Journal of Intelligent Systems, Vol. 8, 1993, p. 749-769, 1993.

[Berglund et al., 1995] Berglund et.al, The use of CAMS during a safety exercise at the Swedish Nuclear Inspectorate, HWR-423. OECD Halden Reactor project -July 1995

[Bowman et al., 1992] Bowman, C.D., Arthur, E.D., Lisowski, P.W., Lawrence, G.P., Jensen, R.J., Anderson, J.L., Blind, B., Cappiello, M., Davidson, J.W., England, T.R., Engel, L.N., Haight, R.C., Hughes, H.G., Ireland, J.R., Krakowski, R.A., LaBauve, R.J., Letellier, B.C., Perry, R.T., Russell, G.J., Staudhammer, K.P., Versamis, G., Wilson, W.B., Nuclear energy generation and waste transmutation using an accelerator-driven intense thermal neutron source. Nucl. Instr. Meth. Phys. Res. A 320, 336, 1992.

[Cammi et al., 2006] Cammi, A., Luzzi, L., Porta, A. A. and Ricotti, M. E., Modelling and control strategy of the Italian LBE-XADS, Progress in Nuclear Energy, Volume 48, Issue 6, Pages 578589, 2006.

[Campolucci et al., 1999] Campolucci, P., Uncini, A., Piazza, F. and Rao, B.D., On-Line Learning Algorithms of Locally Recurrent Neural Networks, IEEE Trans. Neural Networks, 10, pp. 253$271,1999$.

[Carminati et al., 1993] Carminati, F., Klapisch, R., Revol, J.P., Roche, Ch., Rubio, J.A., Rubbia, C., An Energy Amplifier for Cleaner and Inexhaustible Nuclear Energy Production Driven by a Particle Beam Accelerator. CERN Report CERN-AT-93-47(ET), 1993. 
[Chiang et al., 2001] Chiang L. H., Russel, E., Braatz R., Fault detection and diagnosis in industrial systems, Springer-Verlag, London, 2001.

[Dubois et al., 1988] Dubois, D., Prade, H., Testemale, C., Weighted Fuzzy Pattern Matching. Fuzzy Sets and Systems, 28, 1988, p. 313-331, 1988.

[EC, 1999] EC, EU research in Reactor safety: Achievements of the 4th and prospects for the 5Th Euratom framework programme. Eurocourse 1999. GRS, May 1999.

[Glasstone et al., 1998] Glasstone, S, Sesonske, A., Nuclear reactor engineering. New Delhi: CBS Publishers and Distributors; 1998.

[IAEA, 2003] IAEA, Application of simulation techniques for accident management training in nuclear power plants, ISBN: 9201039034, 2003

[Joentgen et al., 1999] Joentgen, A., Mikenina, L., Weber, R., Zimmermann, H.-J., Dynamic Fuzzy Data Analysis based on Similarity between Functions. Fuzzy Sets and Systems, 105 (1), 1999, p. 81-90, 1999.

[Marseguerra et al., 2006] M. Marseguerra, E. Zio, P. Baraldi, I. C. Popescu, and P. Ulmeanu, A Fuzzy Logic - based Model for the Classification of Faults in the Pump Seals of the Primary Heat Transport System of a Candu 6 Reactor, Nuclear Science and Engineering, vol. 153, no. 2, pp. 157-171, 2006.

[NEA, 1992] NEA, Severe Accident Management - Prevention and Mitigation., Paris, 1992

[Øwre, 2001] Øwre, F., Role of the man-machine interface in accident management strategies, Nuclear Engineering and Design 209, 201-210, 2001

[Peel et al., 2008] Peel, L., Data Driven Prognostics using a Kalman Filter Ensemble of Neural Network Models, International Conference on Prognostics and Health Management, 2008.

[Reifman, 1997] J. Reifman, Survey of Artificial Intelligence Methods for Detection and Identification of Component Faults in Nuclear Power Plants, Nuclear Technology., vol. 119, no. 1, pp. 76-97, 1997.

[Rubbia et al., 1995] Rubbia, C., Rubio, J.A., Buono, S., Carminati, F., Fitier, N., Galvez, J., Gels, C., Kadi, Y., Klapisch, R., Mandrillon, P., Revol, J.P., Roche, Ch., Conceptual Design of a Fast Neutron Operated High Power Energy Amplifier. CERN Report CERN-AT-95-44(ET), 1995.

[Santosh et al., 2009] Santosh, T.V., Srivastava, A., Sanyasi Rao, V.V.S., Gosh, A. K., Kushwaha, H.S., Diagnostic System for Identification of Accident Scenarios in Nuclear Power Plants using Artificial Neural Networks, Reliability Engineering and System Safety, 94, 759-762, 2009.

[Serrano et al., 1999] Serrano et al., Development of an extension of the CAMS system to severe accident management, HWR-580. OECD Halden Reactor project -May 1999

[USNRC, 1999] USNRC 1999, NUREG-0700, Rev.1 - Human-System Interface Design Review Guideline, USNRC, Washington, June 1996

[Van Tuyle et al., 1993] Van Tuyle, G.J., Todosow, M., Geiger, M.J., Aronson, A.L., Takahashi, H., Accelerator-driven subcritical target concept for transmutation of nuclear wastes. Nucl. Technol. 101, 1, 1993.

[Venneri et al., 1993] Venneri, F., Bowman, C.D., Jameson R., Accelerator-driven Transmutation of Waste (ATW) - A New Method for Reducing the Long-term Radioactivity of Commercial Nuclear Waste. Los Alamos Report LA-UR-93-752, 1993

[Wang et al., 2008] Wang T., Yu, J., Siegel, D., Lee, J., A similarity based prognostic approach for Remaining Useful life estimation of Engineered Systems, International Conference on Prognostics and Heath Management, 2008.

[Wang et al., 2004] Wang, W.Q., Goldnaraghi, M.F., Ismail, F., Prognosis of Machine Health Condition using Neuro-Fuzzy Systems, Mechanical Systems and Signal Processing, 18, 813831, 2004.

[Willsky, 1976] A. S. Willsky, A Survey of Design Methods for Failure Detection in Dynamic Systems, Automatica, vol. 12, pp. 601-611, 1976. 
[Yuan et al., 1997] Yuan, B., Klir, G., Data driven identification of key variables, In: Ruan, D. (Ed.), Intelligent Hybrid Systems Fuzzy Logic, Neural Network, and Genetic Algorithms. Kluver Academic Publishers, pp.161-187, 1997.

[Zadeh, 1965] Zadeh, L., Fuzzy sets, Inform. Control 8, 338-353, 1965.

[Zio et al., 2005] Zio, E., Baraldi, P., Identification of nuclear transients via optimized fuzzy clustering, Annals of Nuclear Energy, 32, 1068-1080, 2005.

[Zio et al., 2008] Zio, E., Pedroni, N., Broggi, M., Golea, L., Locally recurrent neural networks for nuclear dynamics modeling, FLINS - The 8th International FLINS Conference on Computational Intelligence in Decision and Control, 21-24 September 2008, Madrid, Spain, 2008.

[Zio et al., 2009a] Zio, E., Di Maio, F., A Data Driven Fuzzy Approach for predicting the Remaining recovery Time in a Dynamic Fault Scenarios of a Nuclear System, Reliability Engineering and System Safety, RESS, 10.1016/j.ress.2009.08.001, 2009.

[Zio et al., 2009b] Zio, E., Di Maio, F., Data-Driven on-line prediction of the Available Recovery Time in Nuclear Power Plant Failure Scenarios, submitted to Journal of Risk Analysis, under review process.

[Zio et al., 2009c] Zio, E., Di Maio, F., Processing Dynamic Scenarios from a Reliability Analysis of a Nuclear Power Plant Digital Instrumentation and Control System, Annals of Nuclear Energy, doi:10.1016/j.anucene.2009.06.012, 2009. 